\title{
The cyclooxygenase-2 inhibitor NS-398 inhibits proliferation and induces apoptosis in human osteosarcoma cells via downregulation of the survivin pathway
}

\author{
DA-PENG DUAN ${ }^{1}$, XIAO-QIAN DANG ${ }^{1}$, KUN-ZHENG WANG $^{1}$, \\ YONG-PING WANG ${ }^{2}$, HUI ZHANG ${ }^{3}$ and WU-LIN YOU ${ }^{1}$ \\ ${ }^{1}$ Department of Orthopedics, Second Affiliated Hospital, Xi'an Jiaotong University, Xi'an 710004, Shaanxi Province;
${ }^{2}$ Department of Orthopaedics, Sixth People's Hospital of Shanghai JiaoTong University, Shanghai 200233;
${ }^{3}$ Department of Orthopaedics, Gansu Provincial People's Hospital, Lanzhou 730000, Gansu Province, P.R. China
}

Received April 25, 2012; Accepted July 23, 2012

DOI: 10.3892/or.2012.1992

\begin{abstract}
Cyclooxygenase-2(COX-2) is frequently overexpressed in human malignancies and plays a crucial role in tumorigenesis and cancer progression. The present study aimed to investigate the expression and clinical significance of COX-2 and survivin (SUV) in human osteosarcomas (OS), and explore the effects and molecular mechanisms of a selective COX-2 inhibitor NS-398 and SUV on tumor proliferation and apoptosis. Fifty cases of human OS and osteochondromas (OC) were collected. The expression of COX-2 and SUV was assessed using immunohistochemical assays in biopsy samples. MG-63 human OS cells were treated with different concentrations of NS-398, used to investigate their effects on cell proliferation and apoptosis. The recombinant small hairpin RNA adenovirus vector rAd5-SUV was constructed, and the effects and molecular mechanisms of knockdown of SUV on proliferation and apoptosis were evaluated in MG-63 cells. A subcutaneous xenograft tumor model was established, validating the effects of rAd5-SUV on tumor growth in vivo. Based on the results, the expression of COX-2 and SUV in OS showed a higher strong reactivity rate compared with OC (73.3 vs. $25.0 \%, \mathrm{P}=0.001 ; 63.3$ vs. $30.0 \%, \mathrm{P}=0.02$ ), but it did not correlate with the clinicopathological characteristics of OS. NS-398 inhibited proliferation, induced apoptosis and decreased the mRNA expression of COX-2 and SUV in MG-63 cells. Furthermore, adenovirus-mediated knockdown of SUV inhibited proliferation, induced apoptosis, reduced the expression of proliferating cell nuclear antigen (PCNA), increased the expression of caspase-3 (CAS-3) and slowed the growth of xenograft tumors in MG-63 cells. Taken together, the expression of COX-2
\end{abstract}

Correspondence to: Professor Xiao-Qian Dang, Department of Orthopaedics, Second Affiliated Hospital, Xi'an Jiaotong University, 157 Xiwu Road, Xi'an 710004, Shaanxi Province, P.R. China E-mail: dang_xiaoqian@163.com

Key words: cyclooxygenase-2, NS-398, survivin, osteosarcoma, proliferation, apoptosis and SUV is closely correlated with human OS, and inhibition of COX-2 or knockdown of SUV suppresses tumor proliferation and induces apoptosis, suggesting that $\mathrm{COX}-2$ may be involved in OS cell proliferation and apoptosis through SUV-mediated regulation of PCNA and CAS-3 expression, and provides a potential therapeutic strategy for the treatment of cancer.

\section{Introduction}

Osteosarcoma (OS) is a malignant bone tumor that typically occurs in children, adolescents and young adults. Incorporation of chemotherapy into initial treatment significantly increases the cure rate. However, $\sim 40 \%$ of patients still die from lung metastases $(1,2)$. So, it is very important to develop biomarkers that can inform therapy and provide prognostic insight, especially into identifying poor prognosis patients who should be offered more aggressive therapy at an early time-point in the clinical continuum $(3,4)$. Cancer is also a genetic disease developing from a multi-step process. Single or multiple mutations in genes related to growth control, invasion and metastasis form the molecular genetic basis of malignant transformation and tumor progression (5). Therefore, identification of key genes and targets related to tumorigenesis is crucial for OS prevention and treatment.

Cyclooxygenase-2 (COX-2) is an enzyme catalyzing the conversion of arachidonic acid and $\mathrm{O}_{2}$ to prostaglandin $\mathrm{H}_{2}$, the committed step in prostanoid biosynthesis. The major final end product is prostaglandin $\mathrm{E}_{2}\left(\mathrm{PGE}_{2}\right)$. COX-2 expression is induced by various stimuli, and the overexpression is closely related to the pathogenesis of some degenerative diseases including cancer (6). COX-2 expression is found increased in metastatic rhabdomyosarcoma, leiomyosarcoma and OS, and can be considered as a prognostic value and a target for adjuvant therapy (7-9). COX-2 is highly expressed in high grade OS and application of COX-2 inhibitors may improve the tumor response to chemotherapy and the outcome of OS patients (10). Moreover, COX-2 is directly associated with the proliferation, migration and invasion in human OS cells, and the therapeutic value of COX-2 inhibitors should be evaluated continuously (11). COX-2 expression correlates inversely with disease-specific survival in 
patients with OS lung metastases, indicating that COX-2 expression in metastatic OS may have prognostic significance (12).

Intriguingly, researchers hold different views towards the prognosis of COX-2 in OS. COX-2 overexpression in the primary tumor correlates with the occurrence of distant metastasis in patients with OS, predicts post-metastatic survival and can be taken into consideration in the treatment of patients with OS. COX 2 is a valuable diagnostic marker for OS (13-15). However, it has been proven that there is no significant relationship between COX-2 expression and clinical outcome (16). COX-2 expression does not correlate with outcome of OS or rhabdomyosarcoma (17).

Therefore, it is indispensible to further elucidate the function and molecular regulatory mechanisms of COX-2 in OS. In the present study, the expression and clinical significance of COX-2 and SUV were assessed using immunohistochemical (IHC) assay in biopsy samples. Human MG-63 OS cells were treated with different concentrations of NS-398, used to investigate its effects on cell proliferation and apoptosis. Recombinant small hairpin RNA adenovirus vector rAd5-SUV was constructed, and the effects and molecular mechanisms of knockdown of SUV on proliferation and apoptosis were evaluated in MG-63 cells, attempting to find the potential therapeutic target for the treatment of OS.

\section{Materials and methods}

Materials. MG-63 cell line used in the experiment was from the Laboratory of Second Affiliated Hospital of Xi'an Jiaotong University; 6-week-old female immune-deficient nude mice (BALB/c-nu) were purchased from Shanghai SLAC Laboratory Animal Co., Ltd. (Shanghai Laboratory Animal Center of Chinese Academy Sciences). Adenovirus-mediated SUV small hairpin RNA vector, negative control vector and virion-packaging elements were from Genechem (Shanghai, China); the primers of COX-2, SUV, PCNA and CAS-3 were synthesized by ABI Co., Ltd. (USA). All antibodies were from Santa Cruz Biotechnology (Santa Cruz, CA, USA).

Drugs and reagents. NS-398 was purchased from Cayman Co., Ltd. (USA); 3-(4,5)-dimethylthiahiazo(-z-yl)-3,5-di-phenytetrazoliumbromide (MTT) was from Dingguo Biology (Shanghai, China); Dulbecco's modified Eagle's medium (DMEM) and fetal bovine serum (FBS) were from Thermo Fisher Scientific Inc. (Waltham, MA, USA); TRIzol reagent and Lipofectamine 2000 were from Invitrogen (Carlsbad, CA, USA); M-MLV Reverse Transcriptase was from Promega (Madison, WI, USA); SYBR Green Master Mixture was from Takara (Otsu, Japan); Cell Cycle Analysis kit and apoptosis kit [propidium iodide (PI), RNase A, Annexin V-FITC] were from KeyGEN Biology (Nanjing, China). ECL-PLUS/kit was from GE Healthcare (Piscataway, NJ, USA).

Tissue samples. Fifty freshly resected OS and OC samples were collected at the Department of Orthopedics of Second Affiliated Hospital of Xi'an Jiaotong University during 2010 and were classified according to American Joint Committee on Cancer (AJCC) TNM staging system. Tissues and clinical information were obtained as part of an approved study at Xi'an Jiaotong University. There were 30 cases of OS tissues and 20 cases of OC tissues. A portion of each tissue sample was stored in liquid nitrogen for histopathological and IHC examination. All tumors and normal tissues were diagnosed by two independent pathologists.

IHC staining. Formalin-fixed tissue samples were prepared as paraffin-embedded sections and stained with hematoxylin and eosin. Unstained sections were deparaffinized and incubated overnight at $4{ }^{\circ} \mathrm{C}$ with primary antibodies against COX-2 and SUV and with biotinylated secondary antibody at room temperature for $1 \mathrm{~h}$, followed by incubation with ABC peroxidase and 3,3'-diaminobenzidine (DAB; $30 \mathrm{mg}$ dissolved in $100 \mathrm{ml}$ Tris-buffer containing $0.03 \% \mathrm{H}_{2} \mathrm{O}_{2}$ ). Sections were counterstained with hematoxylin. Expression of COX-2 and SUV in each specimen was scored according to the percentage of positive-stained cells counted in five randomly selected high magnification fields: 0 , no expression; 1 , positive cell ratio $<25 \% ; 2$, positive cell ratio $26-50 \%$; and 3 , positive cell ratio $>50 \%$. The intensity score represents the staining intensity (score 0 , no staining signal; score 1 , weak positive signal; score 2 , moderate positive signal; score 3 , strong positive signal). Finally, a total expression score was given ranging from 0 to 12 . According to the product of these two indicators scoring the results, they were divided into four levels: score $0-2$ is considered as (-), score 3-4 as (+), score 5-7 as (++) and score 8-9 is considered as $(+++)$.

Cell culture and adenovirus transfection. MG-63 cells were cultured in DMEM medium supplemented with $10 \%$ heatinactivated FBS, $100 \mathrm{U} / \mathrm{ml}$ of penicillin and $100 \mu \mathrm{g} / \mathrm{ml}$ of streptomycin. They were all placed in a humidified atmosphere containing $5 \% \mathrm{CO}_{2}$ at $37^{\circ} \mathrm{C}$. Recombinant adenovirus vector rAd5-SUV and negative control rAd5-GFP were transfected into MG-63 cells. Cells were subcultured at a 1:5 dilution in $300 \mu \mathrm{g} /$ $\mathrm{ml}$ G418-containing medium. Positive stable transfectants were selected and expanded for further study. The clone in which the rAd5-SUV virus vectors transfected was named as rAd5-SUV group, the negative control vectors transfected was named as GFP group and MG-63 cells as CON group.

$R T-P C R$. To quantitatively determine the mRNA expression level of COX-2, SUV, PCNA and CAS-3 in MG-63 cells, RT-PCR was used. Total RNA of each clone was extracted with TRIzol according to the manufacturer's instructions. Reverse-transcription was carried out using M-MLV and cDNA amplification was carried out using SYBR Green Master Mix kit according to the manufacturer's instructions. The genes were amplified using specific oligonucleotide primer and human glyceraldehyde-3-phosphate dehydrogenase (GAPDH) gene was used as an endogenous control. The PCR primer sequences were as follows: COX-2, 5'-GAAGTACCAAGCTGT GCTTGAATAA-3' and 5'-GGCTTGATTCCAATGCAC CTA-3'; SUV, 5'-ACCAGGTGAGAAGTGAGGGA-3' and 5'-AACAGTAGAGGAGCCAGGGA-3'; PCNA, 5'-CCATCCT CAAGAAGGTGTTGG-3' and 5'-GTGTCCCATATCCGCAA TTTTAT-3'; CAS-3, 5'-AGAGGGGATCGTTGTAGAAG-3' and 5'-GTTGCCACCTTTCGGTTAAC-3'; GAPDH, 5'-CAAC GAATTTGGCTACAGCA-3' and 5'-AGGGGTCTACATGGC AACTG-3'. Data were analyzed using the comparative $\mathrm{Ct}$ method $\left(2^{-\Delta \Delta C t}\right)$. Three separate experiments were performed for each clone. 
Western blot assay. MG-63 cells were harvested and extracted using lysis buffer (Tris-HCl, SDS, mercaptoethanol, glycerol). Cell extracts were boiled for $5 \mathrm{~min}$ in loading buffer and then equal amount of cell extracts was separated on 15\% SDS-PAGE gels. Separated protein bands were transferred into polyvinylidene fluoride (PVDF) membranes and the membranes were blocked in 5\% skim milk powder. The primary antibodies against COX-2, SUV, PCNA and CAS-3 were diluted according to the instructions of antibodies and incubated overnight at $4^{\circ} \mathrm{C}$. Then, horseradish peroxidase-linked secondary antibodies were added at a dilution ratio of 1:1000, and incubated at room temperature for $2 \mathrm{~h}$. The membranes were washed with PBS three times and the immunoreactive bands were visualized using ECL-PLUS/Kit according to the kit's instruction. The relative protein level in different cell lines was normalized to GAPDH concentration. Three separate experiments were performed for each clone.

Cell proliferation assay. Cell proliferation was analyzed with the MTT assay. Briefly, cells infected with rAd5-SUV were incubated in 96-well-plates at a density of $1 \times 10^{5}$ cells per well with DEME medium supplemented with $10 \%$ FBS. Cells were treated with $20 \mu \mathrm{l}$ MTT dye at $0,24,48$ and $72 \mathrm{~h}$ and then incubated with $150 \mu \mathrm{l}$ of DMSO for $5 \mathrm{~min}$. The color reaction was measured at $570 \mathrm{~nm}$ with enzyme immunoassay analyzer (Bio-Rad, USA). The proliferation activity was calculated for each clone.

Cell apoptosis analysis. To detect cell apoptosis, cells were trypsinized, washed with cold PBS and resuspended in binding buffer according to the instruction of the apoptosis kit. FITCAnnexin V and PI were added to the fixed cells for $20 \mathrm{~min}$ in darkness at room temperature. Then, Annexin $V$ binding buffer was added to the mixture before the fluorescence was measured on FACsort flow cytometer. The cell apoptosis was analyzed using the CellQuest software (Becton-Dickinson, USA). Three separate experiments were performed for each clone.

In vivo tumor xenograft studies. Four mice were injected subcutaneously with $1 \times 10^{8}$ MG-63 cells in $50 \mu 1$ of PBS pre-mixed with an equal volume of matrigel matrix (BectonDickinson). Mice were monitored daily, and three out of four mice developed a subcutaneous tumor. When the tumor size reached approximately $5 \mathrm{~mm}$ in length, they were surgically removed, cut into $1-2 \mathrm{~mm}^{3}$ pieces, and re-seeded individually into 18 other mice. When tumor size reached $\sim 5 \mathrm{~mm}$ in length, the mice were randomly assigned to MG-63, rAd5-GFP and rAd5-SUV groups. In rAd5-GFP and rAd5-SUV groups, $15 \mu \mathrm{l}$ of adenovirus was injected into subcutaneous tumors using a multi-site injection format. Mice in the MG-63 group received $15 \mu 1$ of PBS only. Injections were repeated on the third day after initial treatment. The tumor volume every three days was measured with a caliper, using the formula volume $=$ (length $\mathrm{x}$ width) $)^{2} / 2$.

Statistical analysis. The results of each experiment are shown as mean \pm SD when applicable. Statistically significant difference in each assay was determined by SPSS version 11.5. Difference in each group was tested for significance using

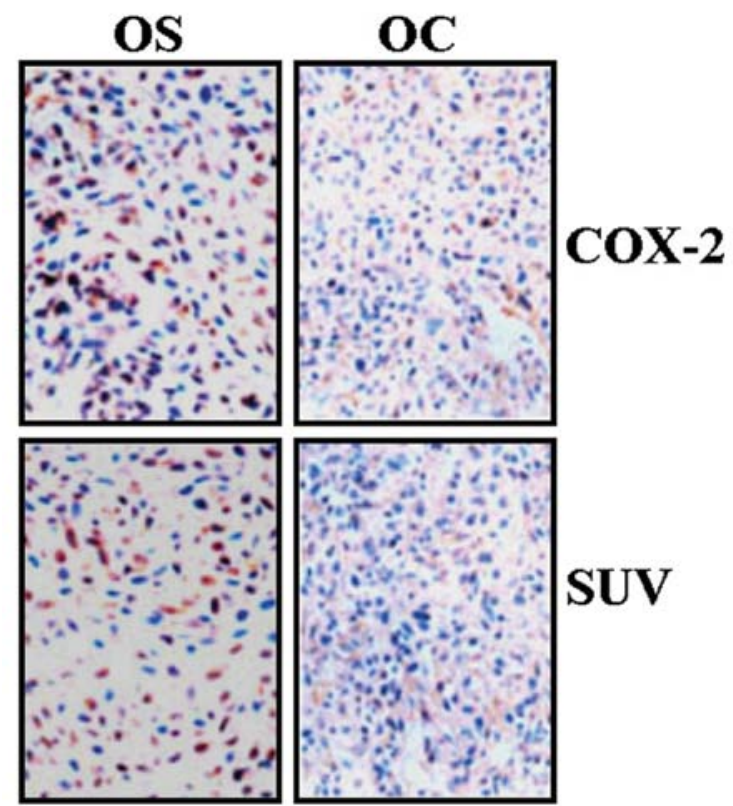

Figure 1. The expression of COX-2 and SUV in human OS. The expression of COX-2 and SUV in OS and OC was evaluated using IHC assays (x400). The results showed that the expression of COX-2 and SUV was mainly localized in the cytoplasm, and was, respectively, observed in 73.3 and $25.0 \%$ OS tissues and in 63.3 and $30.0 \%$ OC tissues, indicating their higher expression in OS compared with OC.

Table I. The expression of COX-2 and SUV in OS and OC tissues.

\begin{tabular}{lcrrrrrrr}
\hline & & \multicolumn{2}{c}{$\mathrm{n}$} & & & & \\
\cline { 3 - 6 } Target & Sample & - & + & Total & $\begin{array}{c}\text { Positive } \\
\text { rate }(\%)\end{array}$ & $\chi^{2}$ & P-value \\
\hline COX-2 & OS & 8 & 22 & 30 & 73.3 & 11.06 & 0.001 \\
& OC & 15 & 5 & 20 & 25.0 & & \\
SUV & OS & 11 & 19 & 30 & 63.3 & 5.23 & 0.02 \\
& OC & 14 & 6 & 20 & 30.0 & & \\
\hline
\end{tabular}

$\chi^{2}$ test and ANOVA analysis of variance. $\mathrm{P}<0.05$ was considered significant.

\section{Results}

The expression of COX-2 and SUV in human OS. The expression of COX-2 and SUV in OS and OC was evaluated using IHC assays. As shown in Fig. 1 and Table I, the expression of COX-2 and SUV was mainly localized in the cytoplasm. The expression of COX-2 and SUV was respectively observed in 73.3 and $63.3 \%$ OS tissues and in 25.0 and $30.0 \%$ OC tissues, indicating their higher expression in OS compared with OC. Spearman rank correlation analysis showed a positive correlation between COX-2 and SUV expression in OS $(r=0.975$, $\mathrm{P}=0.025$ ). 
Table II. The relationship of COX-2 and SUV expression with clinicopathological characteristics of OS.

\begin{tabular}{|c|c|c|c|c|c|c|c|c|}
\hline \multirow[b]{2}{*}{ Clinicopathologic factors } & \multicolumn{2}{|c|}{$\mathrm{COX}-2$} & \multirow[b]{2}{*}{$\chi^{2}$} & \multirow[b]{2}{*}{ P-value } & \multicolumn{2}{|c|}{ SUV } & \multirow[b]{2}{*}{$\chi^{2}$} & \multirow[b]{2}{*}{ P-value } \\
\hline & - & + & & & - & + & & \\
\hline \multicolumn{9}{|l|}{ Age } \\
\hline$>60$ & 5 & 7 & 2.24 & 0.14 & 6 & 7 & 0.86 & 0.35 \\
\hline$<60$ & 3 & 15 & & & 5 & 12 & & \\
\hline \multicolumn{9}{|l|}{ Pathological grade } \\
\hline I & 2 & 5 & & & 3 & 5 & & \\
\hline II & 4 & 10 & 0.13 & 0.94 & 5 & 8 & 0.06 & 0.97 \\
\hline III & 2 & 7 & & & 3 & 6 & & \\
\hline \multicolumn{9}{|l|}{ Pathological type } \\
\hline Osteoblastic & 4 & 9 & & & 4 & 8 & & \\
\hline Chondroblastic & 2 & 8 & 0.34 & 0.84 & 4 & 6 & 0.10 & 0.95 \\
\hline Others & 2 & 5 & & & 3 & 5 & & \\
\hline
\end{tabular}

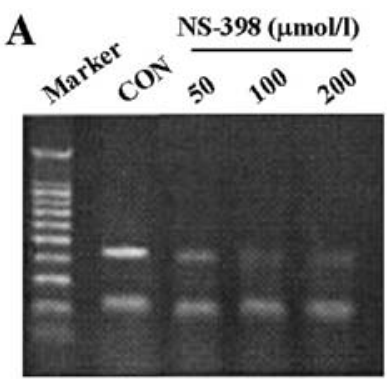

$\operatorname{cox}-2$

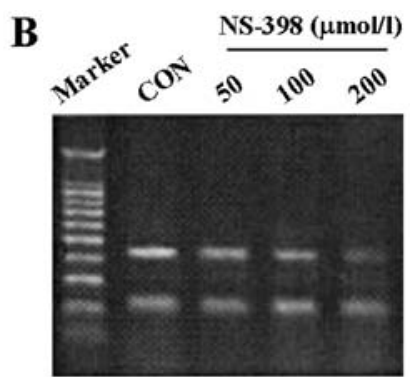

SUV

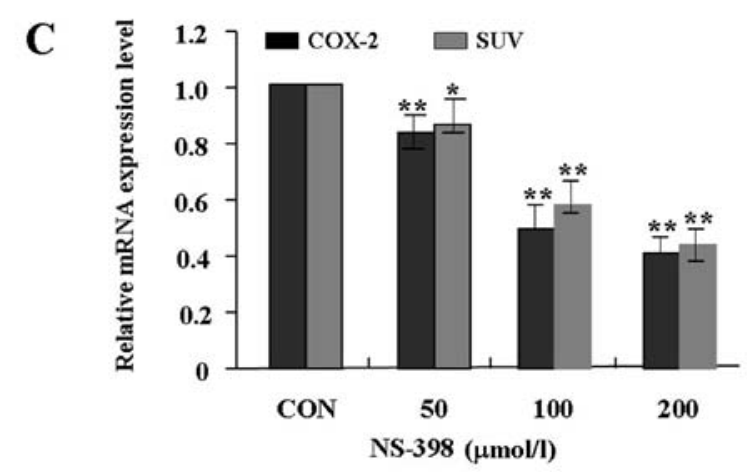

Figure 2. Effects of NS-398 on mRNA expression of COX-2 and SUV in MG-63 cells. (A and B) To examine the effects of NS-398 on expression of COX-2 and SUV in MG-63 cells, MG-63 cells were treated with different concentrations of NS-398 (0, 50, 100 and $200 \mu \mathrm{mol} / \mathrm{l})$. RT-PCR was performed at 48-h recovery to measure their mRNA expression levels. (C) The mRNA expression levels of COX-2 and SUV were significantly lower in NS-398 treated groups in a dose-dependent manner than the control group, suggesting that NS-398 inhibited the mRNA expression of COX-2 and SUV in MG-63 cells $\left({ }^{*} \mathrm{P}<0.05{ }^{* *} \mathrm{P}<0.01\right)$.

The relationship of $C O X-2$ and $S U V$ expression with the clinicopathologic features of $O S$. The relationship between the expression of COX-2 and SUV and clinicopathologic features was analyzed. As shown in Table II, no significant correlation
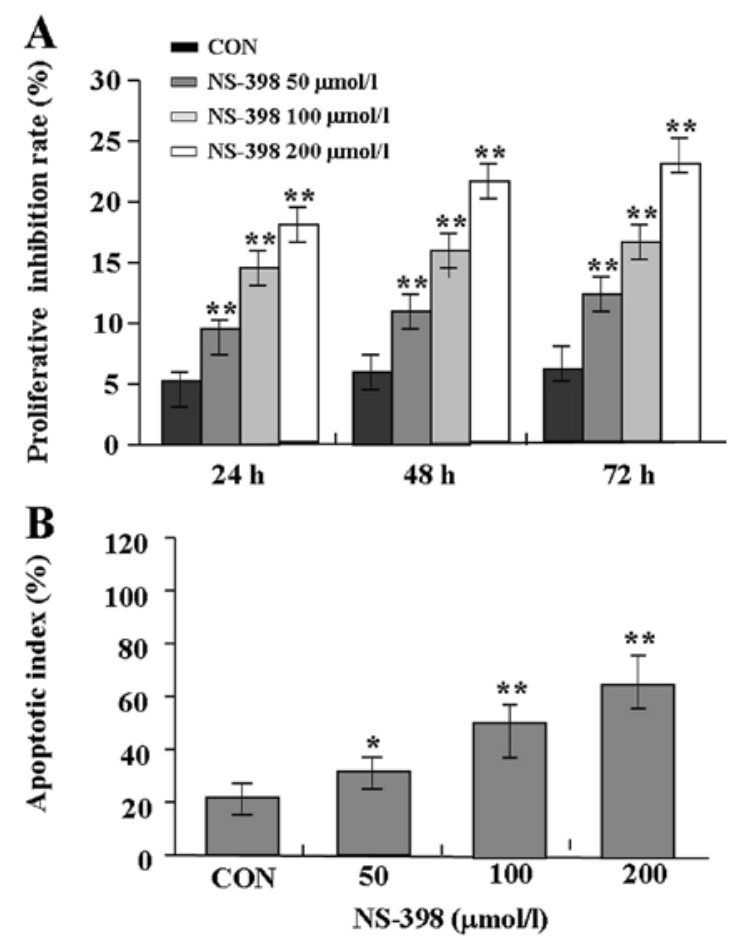

Figure 3. Effects of NS-398 on proliferation and apoptosis of MG-63 cells (A) The proliferative activities of MG-63 cells treated with NS-398 were examined by MTT assay, and it was found that NS-398 could significantly reduce the proliferative activities of MG-63 cells in a dose- and time-dependent manner in comparison with the control group $\left({ }^{* *} \mathrm{P}<0.01\right)$. (B) The apoptotic index of MG-63 cells treated with NS-398 was examined by flow cytometric analysis. The results showed that the apoptosis index of MG-63 cells in NS-398 treated groups was markedly higher than the control group $\left({ }^{*} \mathrm{P}<0.05 ;{ }^{* *} \mathrm{P}<0.01\right)$.

was found between the expression of COX-2 and SUV with age and pathological grade and types of OS.

Effects of NS-398 on mRNA expression of COX-2 and SUV in $M G-63$ cells. To examine the effects of NS-398 on expression of 
A

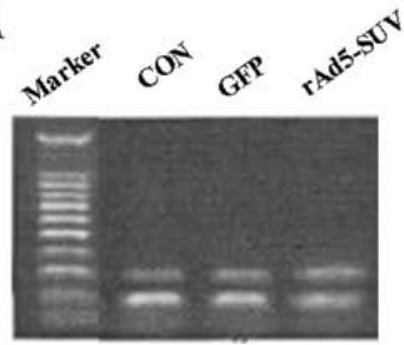

SUV

B

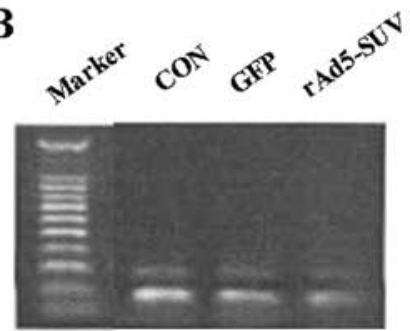

PCNA

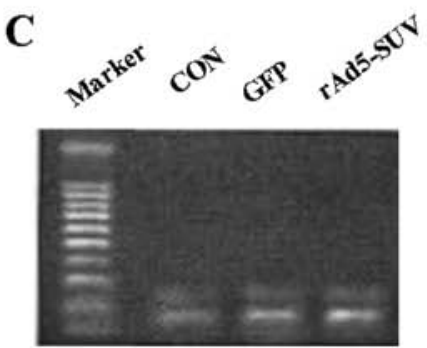

CAS-3
D

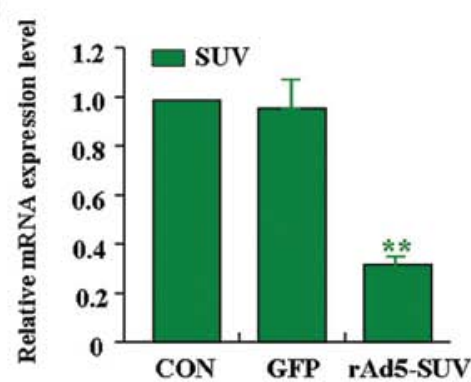

E

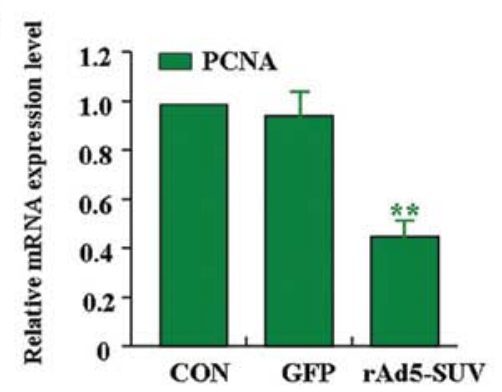

F
G

Fluorescence image

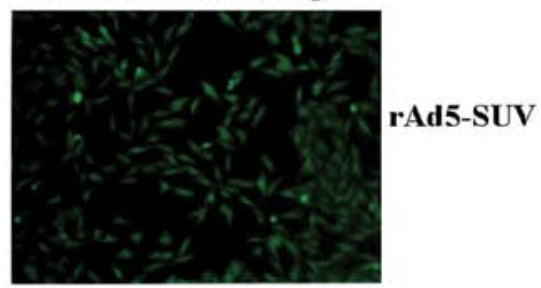

H

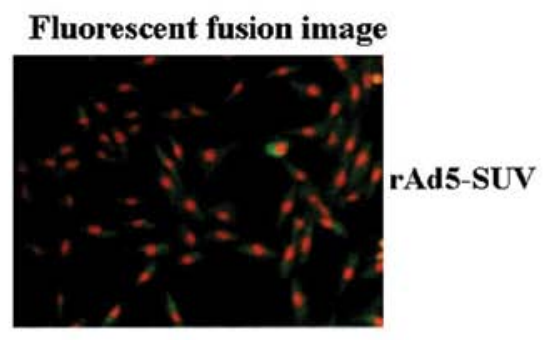

I

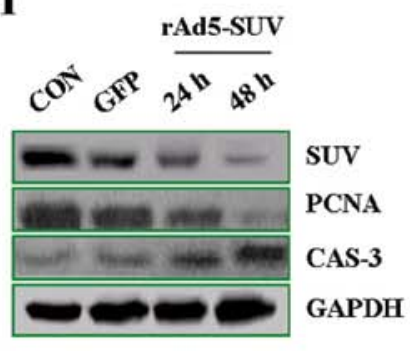

Figure 4. Effects of rAd5-SUV on expression of SUV, PCNA, and CAS-3 in MG-63 cells. (A-C) RT-PCR was performed at 48-h recovery to measure the mRNA expression levels of SUV, PCNA, and CAS-3 in MG-63 cells. (D-F) The mRNA expression of SUV and PCNA was decreased, while CAS-3 expression was increased in rAd5-SUV group compared with the GFP group and CON group ( $\left.{ }^{* *} \mathrm{P}<0.01\right)$. (G and $\left.\mathrm{H}\right)$ An adenovirus-mediated small hairpin RNA approach was used to construct the rAd5-SUV vector. In pilot studies, the transfection efficiency of rAd5-SUV (MOI=100) in MG-63 cells was >95.0\% observed by fluorescence microscopy. (I) Western blotting was performed at 48-h recovery to measure the protein expression levels of SUV, PCNA, and CAS-3 in MG-63 cells. The results demonstrated that the protein expression of SUV and PCNA was decreased, while that of CAS-3 was increased in rAd5-SUV group compared with the GFP group and CON group.

COX-2 and SUV in MG-63 cells, MG-63 cells were treated with different concentrations of NS-398 (0, 50, 100 and $200 \mu \mathrm{mol} / \mathrm{l})$. RT-PCR was performed at 48 -h recovery to measure their mRNA expression levels. As shown in Fig. 2, the mRNA expression levels of COX-2 and SUV were significantly lower in NS-398 treated groups in a dose-dependent manner than the control group, suggesting that NS-398 inhibited the mRNA expression of COX-2 and SUV in MG-63 cells.

Effects of NS-398 on proliferation and apoptosis in MG-63 cells. The proliferative activities of MG-63 cells treated with NS-398 were examined by MTT assay, and it was found that NS-398 could significantly reduce the proliferative activities of MG-63 cells in a dose- and time-dependent manner in comparison with the control group (Fig. 3A). Also, the apoptotic index of MG-63 cells treated with NS-398 was examined by flow cytometric analysis. The results showed that the apoptosis index of MG-63 cells in NS-398 treated groups was markedly higher than the control group (Fig. 3B). Therefore, NS-398 inhibited the proliferation and induced apoptosis in MG-63 cells.
Effects of rAd5-SUV on expression of SUV, PCNA, and CAS-3 in $M G-63$ cells. In order to efficiently knockdown the expression of SUV in MG-63 OS cells, an adenovirus-mediated small hairpin RNA approach was used to construct the rAd5-SUV vector. In pilot studies, the transfection efficiency of rAd5-SUV (MOI=100) in MG-63 cells was $>95.0 \%$ (Fig. 4G and H). After rAd5-SUV was transfected into MG-63 cells, RT-PCR and western blot assays were performed to measure the expression of SUV, PCNA, and CAS-3. As shown in Fig.4A-F and I, the expression of SUV and PCNA was decreased, while CAS-3 expression was increased in rAd5-SUV group compared with the GFP group and CON group. Therefore, knockdown of SUV inhibited the expression of PCNA and enhanced the expression of CAS-3 in MG-63 cells.

Effects of rAd5-SUV on proliferation and apoptosis of MG-63 cells. Deregulated cell proliferation is a hallmark of cancer (18). In order to test the effects of rAd5-SUV on OS cell proliferation and apoptosis, we investigated the proliferative activities and apoptotic index of MG-63 cells by MTT and flow cytometry 


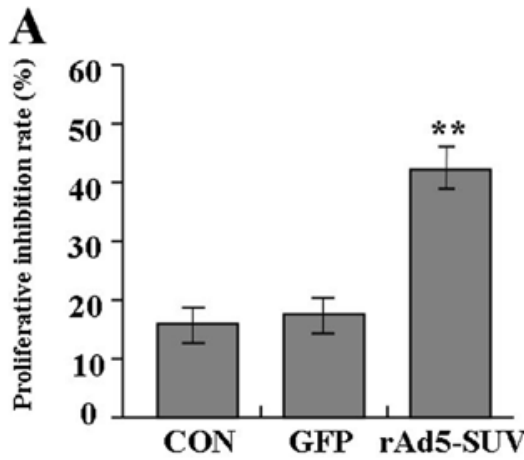

B
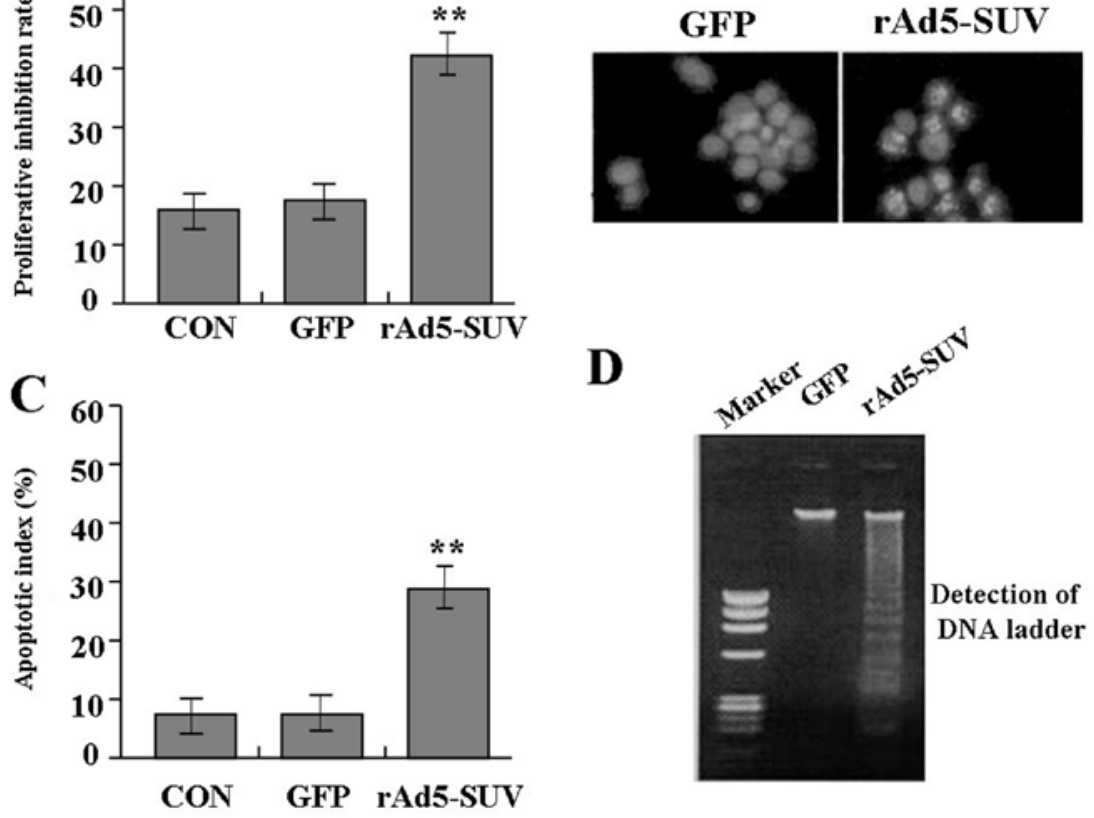

Figure 5. Effects of rAd5-SUV on proliferation and apoptosis of MG-63 cells. (A) The proliferative activities of MG-63 cells were evaluated by MTT analysis. It was indicated that knockdown of SUV could significantly reduce the proliferative activities of MG-63 cells compared with GFP group and CON group $\left({ }^{* *} \mathrm{P}<0.01\right)$. (B and D) Cell nuclear fragmentation, apoptotic bodies and DNA ladder turned up in group rAd5-SUV compared with the group GFP, demonstrating the DNA fragmentation and increase of cell apoptosis induced by knockdown of SUV. (C) Knockdown of SUV markedly increased the apoptotic index of MG-63 cells compared with GFP group and CON group indicated by flow cytometry $\left({ }^{* *} \mathrm{P}<0.01\right)$.
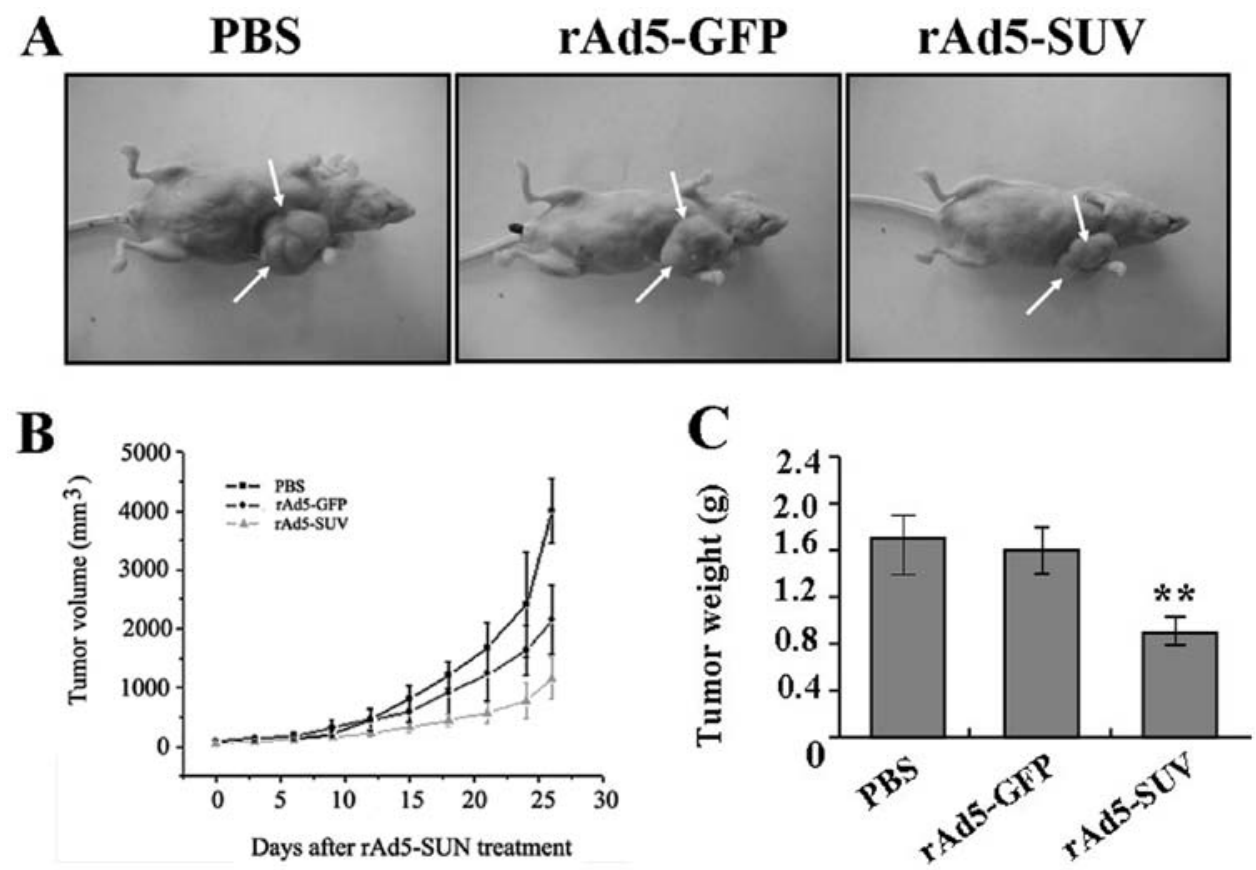

Figure 6. Effects of rAd5-SUV on xenograft tumor growth. (A and B) During the whole tumor growth period, the tumor growth activity was measured. Tumors treated with rAd5-SUV grew substantially slowly compared with the PBS and rAd5-GFP group. (C) When the tumors were harvested, the average weight of tumors in group rAd5-SUV was significantly lower than PBS and rAd5-SUV group $\left({ }^{* *} \mathrm{P}<0.01\right)$.

analysis. As a result, it was indicated that knockdown of SUV could significantly reduce the proliferative activities of MG-63 cells compared with GFP group and CON group (Fig. 5A). Also, cell nuclear fragmentation, apoptotic bodies and DNA ladder turned up in group rAd5-SUV compared with the group GFP, demonstrating the DNA fragmentation and increase of cell apoptosis induced by knockdown of SUV (Fig. 5B and D). Moreover, knockdown of SUV markedly increased the apop- 
totic index of MG-63 cells compared with GFP group and CON group indicated by flow cytometry (Fig. 5C). Therefore, knockdown of SUV inhibited cell proliferation and induce apoptosis in MG-63 cells.

Effects of rAd5-SUV on xenograft tumor growth. Our in vitro experiments demonstrated the inhibitory effects of knockdown of SUV on OS MG-63 cell proliferation. Therefore, it is necessary to further investigate the effect of knockdown of SUV on xenograft tumor growth in vivo. The mean volume of tumors in all experimental mice before treatment was $101.05 \pm 36.27 \mathrm{~mm}^{3}$. During the whole tumor growth period (Fig. 6A and B), the tumor growth activity was measured. Tumors treated with rAd5-SUV grew substantially slowly compared with the PBS and rAd5-GFP group. When the tumors were harvested, the average weight of tumors in group rAd5-SUV was significantly lower than PBS and rAd5-SUV group (Fig. 6C). This result in vivo indicated that knockdown of SUV could also inhibit OS cell growth.

\section{Discussion}

OS is the most frequent malignant bone tumor with a peak incidence in the second and third decade of life. SUV as a member of the inhibitor of apoptosis protein family is expressed both during normal fetal development and in human cancer. Importantly, it is a useful prognostic marker in OS and patients with OS exhibiting nuclear SUV expression could potentially benefit from stratification of neoadjuvant chemotherapy (19). Elevated SUV expression in OS correlates with histologic grade and mitotic index and a decreased disease-free interval. SUV attenuation in canine OS cells inhibits cell cycle progression, increased apoptosis, mitotic arrest and chemosensitivity, and cooperates with chemotherapy to significantly improve tumor control (20). Thus, SUV can be considered as an independent predictor of survival for OS patients $(21,22)$. Coupled with the report that COX-2 expression does not correlate with outcome of OS (16), the relationship of COX-2 and SUV with OS need to be further evaluated. In our study, the expression of COX-2 and SUV was, respectively, observed in 73.3 and $63.3 \%$ OS tissues and in 25.0 and $30.0 \%$ OC tissues, indicating their higher expression in OS than in OC. Spearman rank correlation analysis showed their positive correlation. However, consistent with a previous study (17), our results showed no significant correlation between the expression of COX-2 and SUV with age and pathological grade and type of OS.

In addition, COX-2 inhibitors such as NS-398 and celecoxib have been shown to inhibit COX-2 expression, and produce an anti-proliferative and pro-apoptotic effect on different types of tumor cells $(23,24)$. Meloxicam, the preferential COX-2 inhibitor, inhibits OS growth, invasiveness and metastasis by COX-2-dependent and -independent routes $(25,26)$. RNAImediated knockdown of COX-2 inhibits the growth, invasion and migration of OS, and COX-2 signaling pathway may provide a novel therapeutic target for the treatment of human OS (27). Some data indicate that selective inhibition of COX-2 exerts an effect on primary tumor growth in Ewing sarcoma (28). Furthermore, we investigated the effect of NS-398 on OS cell proliferation and apoptosis, and found that NS-398 significantly inhibited the proliferation and induce apoptosis in OS cells, enriching the anti-tumor evidence of COX-2 inhibitors. Also, the regulatory mechanisms of COX-2 inhibitors on OS are worth exploring. Celecoxib induces apoptosis in human OS cells via downregulation of PI3K/Akt, activating GSK-3 $\beta$ and inhibiting $\beta$-catenin-dependent signaling pathways $(29,30)$. Differently, our study showed that NS-398 downregulated the mRNA expression of SUV in a dose-dependent manner in MG-63 cells, suggesting that MG-63 might inhibit the proliferation and induce apoptosis of MG-63 cells through downregulation of the SUV pathway.

SUV is very important in the development of OS and blockade of SUV markedly inhibits the proliferation and invasion of OS cells, partially reversing their malignant phenotype. Targeting SUV might be a promising option in the treatment of OS and downregulation of SUV is an effective strategy to improve the therapeutic effect of OS (31). Similarly, our study indicated that knockdown of SUV by adenovirus-mediated RNAI inhibited the proliferation, induced apoptosis, and slowed the growth of xenograft tumors in MG-63 cells, providing a strategy for the treatment of OS. PCNA is essential for the replication of deoxyribonucleic acid DNA and has been proved to be an important marker for tumor proliferation. SUV expression has been verified to correlate with PCNA and CAS-3 in OS $(32,33)$. Moreover, we found that knockdown of SUV decreased the expression of PCNA and increased the expression of CAS-3 in MG-63 cells, suggesting that SUV might be involved in OS proliferation and apoptosis via regulation of PCNA and CAS-3 expression.

In conclusion, the expression of COX-2 and SUV is closely correlated with human OS, and NS-398 inhibition of COX-2 or knockdown of SUV by RNAI suppresses tumor proliferation and induces apoptosis in MG-63 cells, suggesting that COX-2 may be involved in OS cell growth and apoptosis through SUV-mediated regulation of PCNA and CAS-3 expression, and provide a potential therapeutic strategy for the treatment of cancer.

\section{References}

1. Jemal A, Bray F, Center MM, et al: Global cancer statistics. CA Cancer J Clin 61: 69-90, 2011.

2. Raymond AK, Ayala AG and Knuutila S: Conventional osteosarcoma. In: World Health Organization Classification of Tumours: Pathology and Genetics of Tumours of Soft Tissue and Bone. Fletcher CDM, Unni KK and Mertens F (eds). IARC Press, Lyon, pp264-270, 2002.

3. Bielack SS, Kempf-Bielack B, Delling G, et al: Prognostic factors in high-grade osteosarcoma of the extremities or trunk: an analysis of 1,702 patients treated on neoadjuvant cooperative osteosarcoma study group protocols. J Clin Oncol 20: 776-790, 2002.

4. Kong $\mathrm{C}$ and Hansen MF: Biomarkers in osteosarcoma. Expert Opin Med Diagn 3: 13-23, 2009.

5. Tajima Y, Yamazaki K, Makino R, et al: Gastric and intestinal phenotypic marker expression in early differentiated-type tumors of the stomach: clinicopathologic significance and genetic background. Clin Cancer Res 12: 6469-6479, 2006.

6. Rodrigues S, Bruyneel E, Rodrigue CM, et al: Cyclooxygenase-2 and carcinogenesis. Bull Cancer 91: S61-S76, 2004.

7. Dickens DS, Kozielski R, Khan J, et al: Cyclooxygenase-2 expression in pediatric sarcomas. Pediatr Dev Pathol 5: 356-364, 2002.

8. Mullins MN, Lana SE, Dernell WS, et al: Cyclooxygenase-2 expression in canine appendicular osteosarcomas. Vet Intern Med 18: 859-865, 2004.

9. Raspollini MR, Amunni G, Villanucci A, et al: Cyclooxygenase-2 expression in uterine leiomyosarcomas. J Chemother 16: 577-581, 2004. 
10. Masi L, Recenti R, Silvestri S, et al: Expression of cyclooxygenase-2 in osteosarcoma of bone. Appl Immunohistochem Mol Morphol 15: 70-76, 2007.

11. Lee EJ, Choi EM, Kim SR, et al: Cyclooxygenase-2 promotes cell proliferation, migration and invasion in U2OS human osteosarcoma cells. Exp Mol Med 39: 469-476, 2007.

12. Rodriguez NI, Hoots WK, Koshkina NV, et al: COX-2 expression correlates with survival in patients with osteosarcoma lung metastases. J Pediatr Hematol Oncol 30: 507-512, 2008.

13. Urakawa H, Nishida Y, Naruse T, et al: Cyclooxygenase-2 overexpression predicts poor survival in patients with high-grade extremity osteosarcoma: a pilot study. Clin Orthop Relat Res 467: 2932-2938, 2009.

14. Boulytcheva IV, Soloviev YN, Kushlinskii NE and Mahson AN Expression of molecular markers in the tumor and survival prognosis in osteosarcoma. Bull Exp Biol Med 150: 237-242, 2010 .

15. Hosono A, Yamaguchi U, Makimoto A, et al: Utility of immunohistochemical analysis for cyclo-oxygenase 2 in the differential diagnosis of osteoblastoma and osteosarcoma. J Clin Pathol 60 410-414, 2007.

16. Carmody Soni EE, Miller BJ, Scarborough MT, et al: Cyclooxygenase- 2 expression is not associated with clinical outcome in synovial sarcoma. Oncol Rep 26: 1513-1517, 2011

17. Dickens DS, Kozielski R, Leavey PJ, et al: Cyclooxygenase-2 expression does not correlate with outcome in osteosarcoma or rhabdomyosarcoma. J Pediatr Hematol Oncol 25: 282-285, 2003

18. Hanahan D and Weinberg RA: The hallmarks of cancer: the next generation. Cell 144: 646-674, 2011.

19. Trieb K, Lehner R, Stulnig T, et al: Survivin expression in human osteosarcoma is a marker for survival. Eur J Surg Oncol 29: 379-382, 2003

20. Shoeneman JK, Ehrhart EJ III, Eickhoff JC, et al: Expression and function of survivin in canine osteosarcoma. Cancer Res 72 249-259, 2012

21. Osaka E, Suzuki T, Osaka S, et al: Survivin expression levels as independent predictors of survival for osteosarcoma patients. J Orthop Res 25: 116-121, 2007.

22. Osaka E, Suzuki T, Osaka S, et al: Survivin as a prognostic factor for osteosarcoma patients. Acta Histochem Cytochem 39: 95-100, 2006.
23. Wiontzek M, Matziolis G, Schuchmann S, et al: Effects of dexamethasone and celecoxib on calcium homeostasis and expression of cyclooxygenase-2 mRNA in MG-63 human osteosarcoma cells. Clin Exp Rheumatol 24: 366-372, 2006.

24. Moalic S,Liagre B, Le Bail JC and Beneytout JL: Dose-dependent modulation of apoptosis and cyclooxygenase-2 expression in human 1547 osteosarcoma cells by NS-398, a selective cyclooxygenase-2 inhibitor. Int J Oncol 18: 533-540, 2001.

25. Naruse T, Nishida Y, Hosono K and Ishiguro N: Meloxicam inhibits osteosarcoma growth, invasiveness and metastasis by COX-2-dependent and independent routes. Carcinogenesis 27: 584-592, 2006.

26. Wolfesberger B, Hoelzl C, Walter I, et al: In vitro effects of meloxicam with or without doxorubicin on canine osteosarcoma cells. Vet Pharmacol Ther 29: 15-23, 2006.

27. Zhao Q, Wang C, Zhu J, et al: RNAi-mediated knockdown of cyclooxygenase 2 inhibits the growth, invasion and migration of SaOS2 human osteosarcoma cells: a case control study. J Exp Clin Cancer Res 30: 26, 2011

28. Gendy AS, Lipskar A, Glick RD, et al: Selective inhibition of cyclooxygenase- 2 suppresses metastatic disease without affecting primary tumor growth in a murine model of Ewing sarcoma. J Pediatr Surg 46: 108-114, 2011.

29. Liu B, Shi ZL, Feng J and Tao HM: Celecoxib, a cyclooxygenase-2 inhibitor, induces apoptosis in human osteosarcoma cell line MG-63 via down-regulation of PI3K/Akt. Cell Biol Int 32: 494-501, 2008

30. Xia JJ, Pei LB, Zhuang JP, et al: Celecoxib inhibits $\beta$-catenindependent survival of the human osteosarcoma MG-63 cell line. J Int Med Res 38: 1294-1304, 2010.

31. Liang X, Da M, Zhuang Z, et al: Effects of Survivin on cell proliferation and apoptosis in MG-63 cells in vitro. Cell Biol Int 33: 119-124, 2009.

32. Wang W, Luo $\mathrm{H}$ and Wang A: Expression of survivin and correlation with PCNA in osteosarcoma. J Surg Oncol 93: 578-584, 2006.

33. Zou J, Gan M, Mao N, et al: Sensitization of osteosarcoma cell line SaOS-2 to chemotherapy by downregulating survivin. Arch Med Res 41: 162-169, 2010. 